\title{
Transverse momentum distributions of hadrons in the Tsallis- 1 and Tsallis-2 statistics
}

\author{
A.S. Parvan ${ }^{1,2,3, \star}$ \\ ${ }^{1}$ Bogoliubov Laboratory of Theoretical Physics, Joint Institute for Nuclear Research, Dubna, Russia \\ ${ }^{2}$ Department of Theoretical Physics, Horia Hulubei National Institute of Physics and Nuclear Engineering, \\ Bucharest-Magurele, Romania \\ ${ }^{3}$ Institute of Applied Physics, Moldova Academy of Sciences, Chisinau, Republic of Moldova
}

\begin{abstract}
We considered the ultrarelativistic transverse momentum distributions of the Tsallis- 1 and Tsallis- 2 statistics using two regularization schemes. It was revealed that the cut-off parameter strongly influences the behavior of the transverse momentum distribution in both statistics. We have also found that the ultrarelativistic transverse momentum distribution of the Tsallis-1 statistics is transformed to the momentum distribution of the Tsallis- 2 statistics by identifying $q \rightarrow 1 / q_{c}$.
\end{abstract}

\section{Introduction}

The transverse momentum distributions of the Tsallis-factorized statistics [1, 2] are largely used to analyze the LHC and RHIC data on the transverse momentum distributions of hadrons created in the proton-proton and heavy-ion collisions. The Tsallis statistics $[3,4]$ was applied to treat the experimental transverse momentum distributions of hadrons only in the ultrarelativistic case and only for the first variant of the Tsallis statistics [5]. In [6] it was demonstrated that the Tsallis-factorized statistics is not equivalent to the Tsallis statistics. In [7] it was found out that the parameter $q$, which indicates the deviation of the Tsallis distribution from the usual Boltzmann distribution, effectively takes into account the hard processes of the partons reactions, which lead to the creation of energetically high$p_{T}$ hadrons. The main purpose of this study is to establish the relation between the cut-off parameter and the behavior of the transverse momentum distributions of the Tsallis- 1 and Tsallis- 2 statistics.

\section{Tsallis-1 statistics for $q<1$}

In the Tsallis- 1 statistics, the ensemble averages, the probabilities of microstates and the norm equation in the grand canonical ensemble are written as follows [8]:

$$
\begin{aligned}
\langle A\rangle & =\sum_{i} A_{i}\left[1+\frac{q-1}{q} \frac{\Lambda-E_{i}+\mu N_{i}}{T}\right]^{\frac{1}{q-1}}, \quad p_{i}=\left[1+\frac{q-1}{q} \frac{\Lambda-E_{i}+\mu N_{i}}{T}\right]^{\frac{1}{q-1}}, \\
1 & =\sum_{i}\left[1+\frac{q-1}{q} \frac{\Lambda-E_{i}+\mu N_{i}}{T}\right]^{\frac{1}{q-1}},
\end{aligned}
$$

^e-mail: parvan@theor.jinr.ru;parvan@theory.nipne.ro 
where $E_{i}$ and $N_{i}$ are the energy and the number of particles in the $i$-th microscopic state of the system, respectively, $\Lambda$ is a norm function and $q \in \mathbf{R}$ is a real parameter taking values $0<q<\infty$.

The norm equation (2) for the Maxwell-Boltzmann ultrarelativistic ideal gas of the Tsallis-1 statistics in the grand canonical ensemble can be written as [6]

$$
\sum_{\left\{n_{\vec{p} \sigma}\right\}} \frac{1}{\prod_{\vec{p} \sigma} n_{\vec{p} \sigma} !}\left[1+\frac{q-1}{q} \frac{\Lambda-\sum_{\vec{p} \sigma} n_{\vec{p} \sigma}\left(\varepsilon_{\vec{p}}-\mu\right)}{T}\right]^{\frac{1}{q-1}}=1,
$$

where $\varepsilon_{\vec{p}}=|\vec{p}|$ is the one-particle energy and $n_{\vec{p} \sigma}=0,1, \ldots, \infty$ are the occupation numbers. Using the integral representations for the Gamma-function [9]

$$
x^{-y}=\frac{1}{\Gamma(y)} \int_{0}^{\infty} t^{y-1} e^{-t x} d t, \quad \operatorname{Re}(x)>0, \operatorname{Re}(y)>0
$$

we obtain

$$
\frac{1}{\Gamma\left(\frac{1}{1-q}\right)} \int_{0}^{\infty} t^{\frac{1}{1-q}-1} e^{-t\left[1+\frac{q-1}{q} \frac{\Lambda}{T}\right]} Z\left(\beta^{\prime}\right) d t=1,
$$

where $\beta^{\prime}=-(q-1) t /(q T)$ and

$$
Z\left(\beta^{\prime}\right)=\sum_{\left\{n_{\vec{p} \sigma}\right\}} \frac{1}{\prod_{\vec{p} \sigma} n_{\vec{p} \sigma} !} e^{-\beta^{\prime} \sum_{\vec{p} \sigma} n_{\vec{p} \sigma}\left(\varepsilon_{\vec{p}}-\mu\right)}=\exp \left(\sum_{\vec{p} \sigma} e^{-\beta^{\prime}\left(\varepsilon_{\vec{p}}-\mu\right)}\right) .
$$

For the ultrarelativistic ideal gas the partition function (6) can be rewritten as

$$
Z\left(\beta^{\prime}\right)=\exp \left(\frac{g V}{\pi^{2} \beta^{\prime 3}} e^{\beta^{\prime} \mu}\right)=\sum_{N=0}^{\infty} \frac{1}{N !}\left(\frac{g V}{\pi^{2} \beta^{\prime 3}}\right)^{N} e^{\beta^{\prime} \mu N},
$$

where $g$ is the spin degeneracy factor. Substituting (7) into (5) and using (4) we have [6]

$$
\sum_{N=0}^{N_{0}} \frac{\tilde{\omega}^{N}}{N !} \frac{\Gamma\left(\frac{1}{1-q}-3 N\right)}{\left(\frac{1-q}{q}\right)^{3 N} \Gamma\left(\frac{1}{1-q}\right)}\left[1+\frac{q-1}{q} \frac{\Lambda+\mu N}{T}\right]^{\frac{1}{q-1}+3 N}=1,
$$

where $\tilde{\omega}=g V T^{3} / \pi^{2}$ and $N_{0}<1 /(3(1-q))$. The terms with $N>N_{0}$ in the series (8) are divergent and they should be excluded. Note that $N$ is not the number of particles.

The mean occupation numbers for the Maxwell-Boltzmann ultrarelativistic ideal gas in the Tsallis1 statistics can be written as [6]

$$
\left\langle n_{\vec{p} \sigma}\right\rangle=\sum_{\left\{n_{\vec{p} \sigma}\right\}} n_{\vec{p} \sigma} \frac{1}{\prod_{\vec{p} \sigma} n_{\vec{p} \sigma} !}\left[1+\frac{q-1}{q} \frac{\Lambda-\sum_{\vec{p} \sigma} n_{\vec{p} \sigma}\left(\varepsilon_{\vec{p}}-\mu\right)}{T}\right]^{\frac{1}{q-1}} .
$$

Using (4), we obtain

$$
\left\langle n_{\vec{p} \sigma}\right\rangle=\frac{1}{\Gamma\left(\frac{1}{1-q}\right)} \int_{0}^{\infty} t^{\frac{1}{1-q}-1} e^{-t\left[1+\frac{q-1}{q} \frac{\Lambda}{T}\right]} Z\left(\beta^{\prime}\right)\left\langle n_{\vec{p} \sigma}\right\rangle_{G} d t
$$


where

$$
\left\langle n_{\vec{p} \sigma}\right\rangle_{G}=\frac{1}{Z\left(\beta^{\prime}\right)} \sum_{\left\{n_{\vec{p} \sigma}\right\}} n_{\vec{p} \sigma} \frac{1}{\prod_{\vec{p} \sigma} n_{\vec{p} \sigma} !} e^{-\beta^{\prime} \sum n_{\vec{p} \sigma}\left(\varepsilon_{\vec{p}}-\mu\right)}=e^{-\beta^{\prime}\left(\varepsilon_{\vec{p}}-\mu\right)} .
$$

Substituting (11) and (7) into (10) and using (4), we obtain [6]

$$
\left\langle n_{\vec{p} \sigma}\right\rangle=\sum_{N=0}^{N_{0}} \frac{\tilde{\omega}^{N}}{N !} \frac{\Gamma\left(\frac{1}{1-q}-3 N\right)}{\left(\frac{1-q}{q}\right)^{3 N} \Gamma\left(\frac{1}{1-q}\right)}\left[1+\frac{q-1}{q} \frac{\Lambda-\varepsilon_{\vec{p}}+\mu(N+1)}{T}\right]^{\frac{1}{q-1}+3 N},
$$

where the upper bound of summation $N_{0}$ is the same as in (8). Then the transverse momentum distribution for the ultrarelativistic particles can be written as [6]

$$
\frac{d^{2} N}{d p_{T} d y}=\frac{g V}{(2 \pi)^{2}} p_{T}^{2} \cosh y \sum_{N=0}^{N_{0}} \frac{\tilde{\omega}^{N}}{N !} \frac{\Gamma\left(\frac{1}{1-q}-3 N\right)}{\left(\frac{1-q}{q}\right)^{3 N} \Gamma\left(\frac{1}{1-q}\right)}\left[1+\frac{q-1}{q} \frac{\Lambda-\varepsilon_{\vec{p}}+\mu(N+1)}{T}\right]^{\frac{1}{q-1}+3 N} .
$$

In the Gibbs limit $q \rightarrow 1$ (13) recovers the Maxwell-Boltzmann transverse momentum distribution of the Boltzmann-Gibbs statistics

$$
\frac{d^{2} N}{d p_{T} d y}=\frac{g V}{(2 \pi)^{2}} p_{T}^{2} \cosh y e^{-\frac{p_{T} \cosh y-\mu}{T}}
$$

Taking the zeroth term $N=0$ in (13) and (8) and transforming $q \rightarrow 1 / q_{c}$ [6], we exactly obtain the transverse momentum distribution of the Tsallis-factorized statistics $[1,2]$

$$
\frac{d^{2} N}{d p_{T} d y}=\frac{g V p_{T}^{2} \cosh y}{(2 \pi)^{2}}\left[1+\left(q_{c}-1\right) \frac{p_{T} \cosh y-\mu}{T}\right]^{\frac{q_{c}}{1-q_{c}}}
$$

To find the upper bound of summation $N_{0}$, we rewrite (8) in the form $\sum_{N} \phi(N)=1$, where

$$
\phi(N)=\frac{\tilde{\omega}^{N}}{N !} \frac{\Gamma\left(\frac{1}{1-q}-3 N\right)}{\left(\frac{1-q}{q}\right)^{3 N} \Gamma\left(\frac{1}{1-q}\right)}\left[1+\frac{q-1}{q} \frac{\Lambda+\mu N}{T}\right]^{\frac{1}{q-1}+3 N} .
$$

In the Gibbs limit $q \rightarrow 1$ (16) recovers the Maxwell-Boltzmann function of the Boltzmann-Gibbs statistics $\phi(N)=\frac{\tilde{\omega}^{N}}{N !} e^{\frac{\Lambda+\mu N}{T}}$, where $\Lambda=-T \tilde{\omega} e^{\frac{\mu}{T}}$.

We have two possibilities to find the cut-off parameter $N_{0}$ in (8). See figure 1 . In the first method of truncation of the series, which we denote as the Model A, we find $N_{0}$ from the local minimum of the function $\ln \phi(N)$ :

$$
\left.\frac{\partial \ln \phi(N)}{\partial N}\right|_{N=N_{0}}=0
$$

However, in the second method of truncation of the series, which we denote as the Model B, we find $N_{0}$ from the inflection point of the function $\ln \phi(N)[6]$ :

$$
\left.\frac{\partial^{2} \ln \phi(N)}{\partial N^{2}}\right|_{N=N_{0}}=0 .
$$

The transverse momentum distribution of the Model A differs essentially from the transverse momentum distributions of the Model B and the Tsallis-factorized statistics due to the large values of the cut-off parameter $N_{0}$. However, the transverse momentum distribution of the Model B is close to the transverse momentum distribution of the Tsallis-factorized statistics, because the values of $N_{0}$ in the Model B are close to zero. 

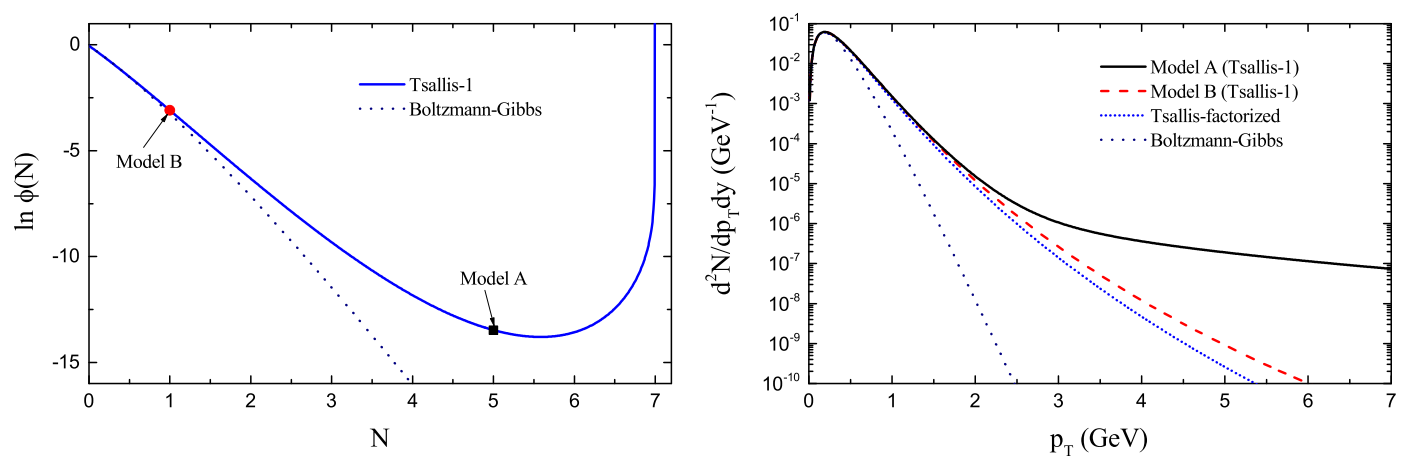

Figure 1. Two methods to fix the cut-off parameter $N_{0}$ in the Tsallis-1 statistics. The behavior of $\ln \phi(N)$ as a function of $N$ (left panel) and the transverse momentum distribution (right panel) for the ultrarelativistic ideal gas of $\pi^{0}$ pions in the Tsallis-1 statistics in the volume $V=1 \mathrm{fm}$ at the temperature $T=90 \mathrm{MeV}$, chemical potential $\mu=0$, rapidity $y=0$ and $q_{c}=1.05\left(q=1 / q_{c}\right)$.

\section{Tsallis- 2 statistics for $q_{c}>1$}

In the Tsallis-2 statistics, the ensemble averages, the probabilities of microstates and the partition function in the grand canonical ensemble are written as follows $[4,6]$ :

$$
\begin{aligned}
\langle A\rangle & =\frac{1}{Z^{q_{c}}} \sum_{i} A_{i}\left[1-\left(1-q_{c}\right) \frac{E_{i}-\mu N_{i}}{T}\right]^{\frac{q_{c}}{1-q_{c}}}, \quad p_{i}=\frac{1}{Z}\left[1-\left(1-q_{c}\right) \frac{E_{i}-\mu N_{i}}{T}\right]^{\frac{1}{1-q_{c}}}, \\
Z & =\sum_{i}\left[1-\left(1-q_{c}\right) \frac{E_{i}-\mu N_{i}}{T}\right]^{\frac{1}{1-q_{c}}},
\end{aligned}
$$

where $Z$ is the partition function and $q_{c} \in \mathbf{R}$ is a real parameter taking values $0<q_{c}<\infty$. Note that $q_{c}$ is a finite number and it cannot be equal to infinity.

The partition function (20) for the Maxwell-Boltzmann ultrarelativistic ideal gas in the framework of the Tsallis-1 statistics in the grand canonical ensemble can be written as [6]

$$
Z=\sum_{N=0}^{N_{0}} \frac{\tilde{\omega}^{N}}{N !} \frac{\Gamma\left(\frac{1}{q_{c}-1}-3 N\right)}{\left(q_{c}-1\right)^{3 N} \Gamma\left(\frac{1}{q_{c}-1}\right)}\left[1+\left(1-q_{c}\right) \frac{\mu N}{T}\right]^{\frac{1}{1-q_{c}}+3 N},
$$

where $N_{0}<1 /\left(3\left(q_{c}-1\right)\right)$. The terms with $N>N_{0}$ in the series (21) are divergent and they should be excluded. Note that $N$ is not the number of particles.

The mean occupation numbers for the Maxwell-Boltzmann ultrarelativistic ideal gas in the Tsallis2 statistics in the grand canonical ensemble for $q_{c}>1$ can be written as [6]

$$
\left\langle n_{\vec{p} \sigma}\right\rangle=\frac{1}{Z^{q_{c}}} \sum_{N=0}^{N_{0}} \frac{\tilde{\omega}^{N}}{N !} \frac{\Gamma\left(\frac{q_{c}}{q_{c}-1}-3 N\right)}{\left(q_{c}-1\right)^{3 N} \Gamma\left(\frac{q_{c}}{q_{c}-1}\right)}\left[1+\left(q_{c}-1\right) \frac{\varepsilon_{\vec{p}}-\mu(N+1)}{T}\right]^{\frac{q_{c}}{1-q_{c}}+3 N},
$$

where the upper bound of summation $N_{0}$ is the same as in (21). Then the transverse momentum distribution for the ultrarelativistic particles can be written as

$$
\frac{d^{2} N}{d p_{T} d y}=\frac{g V}{(2 \pi)^{2}} p_{T}^{2} \cosh y \frac{1}{Z^{q_{c}}} \sum_{N=0}^{N_{0}} \frac{\tilde{\omega}^{N}}{N !} \frac{\Gamma\left(\frac{q_{c}}{q_{c}-1}-3 N\right)}{\left(q_{c}-1\right)^{3 N} \Gamma\left(\frac{q_{c}}{q_{c}-1}\right)}\left[1+\left(q_{c}-1\right) \frac{\varepsilon_{\vec{p}}-\mu(N+1)}{T}\right]^{\frac{q_{c}}{1-q_{c}}+3 N}
$$


In the Gibbs limit $q_{c} \rightarrow 1$, (23) recovers the Maxwell-Boltzmann transverse momentum distribution (14) of the Boltzmann-Gibbs statistics. Taking the zeroth term $N=0$ in (23) and (21), we exactly obtain the transverse momentum distribution (15) of the Tsallis-factorized statistics.

To find the upper bound of summation $N_{0}$, we rewrite the partition function (21) in the form $Z=\sum_{N} \phi(N)$, where

$$
\phi(N)=\frac{\tilde{\omega}^{N}}{N !} \frac{\Gamma\left(\frac{1}{q_{c}-1}-3 N\right)}{\left(q_{c}-1\right)^{3 N} \Gamma\left(\frac{1}{q_{c}-1}\right)}\left[1+\left(1-q_{c}\right) \frac{\mu N}{T}\right]^{\frac{1}{1-q_{c}}+3 N}
$$

In the Gibbs limit $q_{c} \rightarrow 1$ (24) recovers the Maxwell-Boltzmann function of the Boltzmann-Gibbs statistics $\phi(N)=\frac{\tilde{\omega}^{N}}{N !} e^{\frac{\mu N}{T}}$.
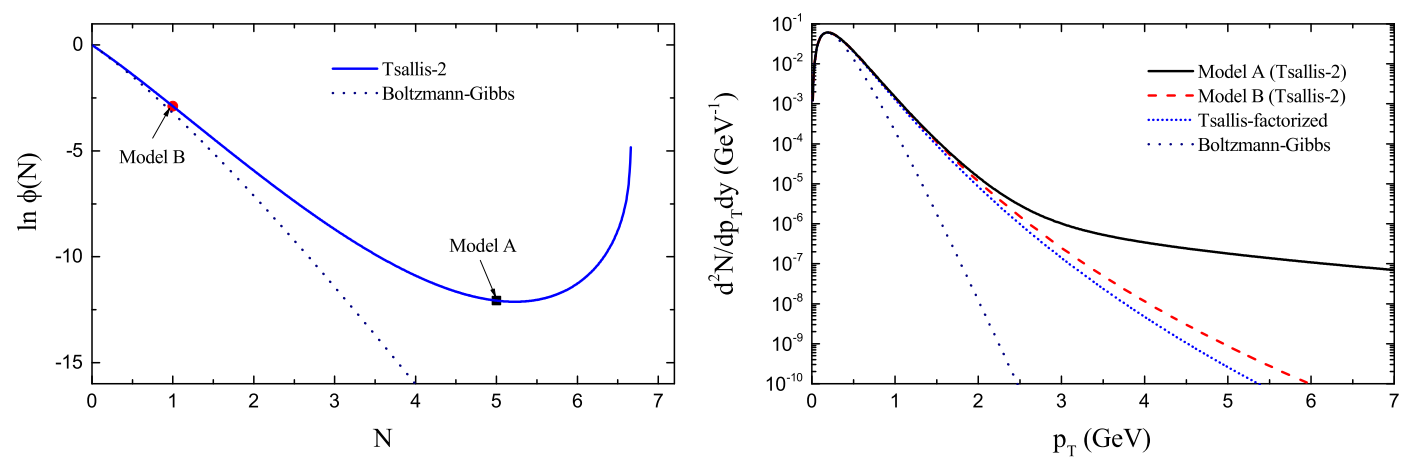

Figure 2. Two methods to fix the cut-off parameter $N_{0}$ in the Tsallis-2 statistics. The behavior of $\ln \phi(N)$ as a function of $N$ (left panel) and the transverse momentum distribution (right panel) for the ultrarelativistic ideal gas of $\pi^{0}$ pions in the Tsallis- 2 statistics. The values of the parameters are the same as in figure 1.

In the Model A, the cut-off parameter $N_{0}$ is found from the local minimum of the function $\ln \phi(N)$ substituting (24) into (17). However, in the Model B, the cut-off parameter $N_{0}$ is found from the inflection point of the function $\ln \phi(N)$ substituting (24) into (18). The results of these two methods are illustrated in figure 2. Thus, in the Tsallis-2 statistics the transverse momentum distribution of the Model A differs essentially from the transverse momentum distribution of the Model B and the Tsallis-factorized statistics due to the large values of the cut-off parameter $N_{0}$. However, the results of the Model B are close to the results of the Tsallis-factorized statistics, because the values of $N_{0}$ in the Model B are close to zero.

\section{Comparison of the Tsallis- 1 and Tsallis- 2 statistics}

In figure 3, we compare the transverse momentum distributions of the Tsallis- 1 statistics with the transverse momentum distributions of the Tsallis- 2 statistics in the Model A and the Model B. The parameter $q$ of the Tsallis- 1 statistics was obtained by the equality $q=1 / q_{c}$. We have obtained that the results of the Tsallis-1 statistics practically coincide with the results of the Tsallis- 2 statistics. Thus, the ultrarelativistic transverse momentum distribution of the Tsallis-1 statistics is transformed to the ultrarelativistic transverse momentum distribution of the Tsallis- 2 statistics by identifying $q \rightarrow 1 / q_{c}$. 

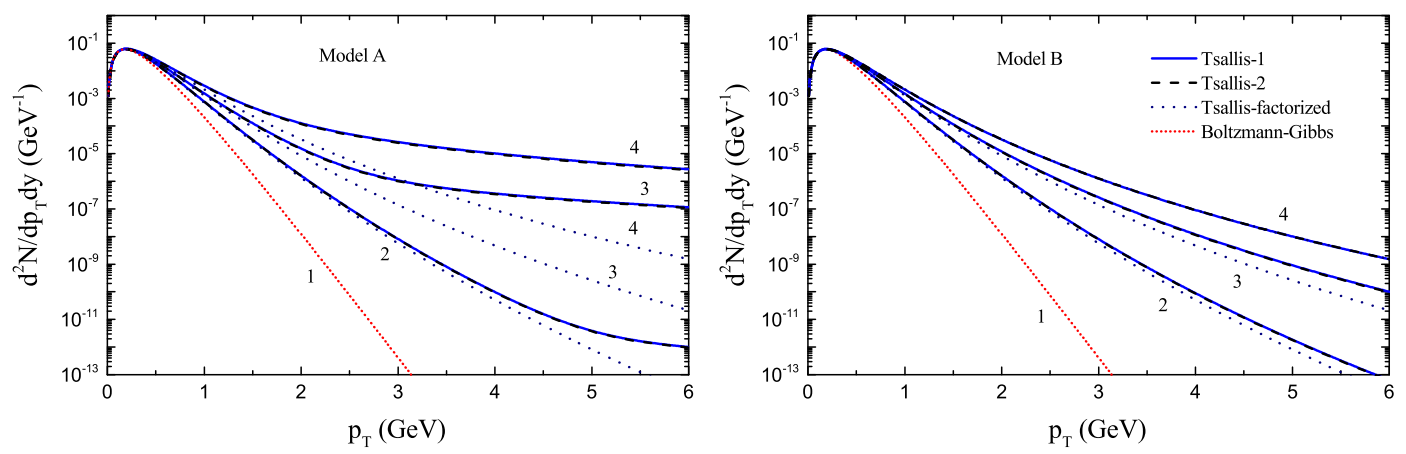

Figure 3. The transverse momentum distribution for the ultrarelativistic ideal gas of $\pi^{0}$ pions in the Model A (left panel) and the Model B (right panel) for the Tsallis-1 and Tsallis-2 statistics in the volume $V=1 \mathrm{fm}$ at the temperature $T=90 \mathrm{MeV}$, chemical potential $\mu=0$, rapidity $y=0$ and different values of $q_{c}\left(q=1 / q_{c}\right)$. The lines $1,2,3$ and 4 correspond to $q_{c}=1,1.03,1.05$ and 1.07 , respectively.

\section{Conclusions}

The analytical formulae for the ultrarelativistic transverse momentum distributions of the Tsallis-1 and Tsallis-2 statistics were derived in detail. To regularize them, we use two cut-off prescriptions: the Model A and the Model B. It was revealed that the cut-off parameter strongly influences the behavior of the transverse momentum distribution in both the Tsallis- 1 and Tsallis- 2 statistics. In the Model A, in which the cut-off parameter is found from the local minimum of the function $\ln \phi(N)$, the transverse momentum distribution differs essentially from the transverse momentum distribution of the Tsallis-factorized statistics. However, in the Model B, in which the cut-off parameter is found from the inflection point of the function $\ln \phi(N)$, the transverse momentum distribution is very close to the transverse momentum distribution of the Tsallis-factorized statistics. We have also found that the ultrarelativistic transverse momentum distribution of the Tsallis- 1 statistics is transformed to the momentum distribution of the Tsallis-2 statistics by identifying $q \rightarrow 1 / q_{c}$.

Acknowledgments: This work was supported in part by the joint research project and grant of JINR and IFIN-HH (protocol N 4543).

\section{References}

[1] J. Cleymans, D. Worku, J. Phys. G: Nucl. Part. Phys. 39, 025006 (2012)

[2] J. Cleymans, D. Worku, Eur. Phys. J. A 48, 160 (2012)

[3] C. Tsallis, J. Stat. Phys. 52, 479 (1988)

[4] C. Tsallis, R.S. Mendes, A.R. Plastino, Physica A 261, 534 (1998)

[5] A.S. Parvan, Eur. Phys. J. A 52, 355 (2016)

[6] A.S. Parvan, arXiv:1607.07670v2 [hep-ph]

[7] A.S. Parvan, O.V. Teryaev, J. Cleymans, arXiv:1607.01956v2 [nucl-th]

[8] A.S. Parvan, Eur. Phys. J. A 51, 108 (2015)

[9] D. Prato, Phys. Lett. A 203, 165 (1995) 